\title{
A FRAMING OF DESIGN AS PATHWAYS BETWEEN PHYSICAL, VIRTUAL AND COGNITIVE MODELS
}

\author{
D. E. Jones ${ }^{凶}$, C. Snider and B. Hicks \\ University of Bristol, United Kingdom \\ $凶$ david.jones@bristol.ac.uk
}

\section{Abstract}

During engineering design, designers employ three types of model: physical, virtual and cognitive. The role and contribution of each is documented in literature albeit fragmented in nature. Consequentially, a gap in understanding exists in terms of how these models and the transitions between them impact the designer and design process. This paper begins to address this through a characterisation of each model class and an appraisal of the transitional pathways including their alignment to seminal design frameworks.

Keywords:early design phase, prototyping, design models

\section{Introduction}

Within the paradigm of the digital twin for early stage design (Jones et al., 2019), it becomes clear that the virtual-to-physical and physical-to-virtual connections of the digital twin fail to account for all elements of early stage design. Questions such as how one 'twins' a design concept, for example, cannot be fully met by the digital twin in its current form, as it neglects the role and importance of the designer. Furthermore, in recent years, work in areas such as generative design (Shea et al., 2005) and attempts to democratise design (Goudswaard et al., 2018) are at risk of "designing out" the designer. While these are interesting scientific endeavours, there is a risk that the designer is being replaced without fully understanding the importance and role that they play or could play in the design process.

In contrast, the Open University's course material on the introduction to design ${ }^{1}$ describes design activities in terms of three types of model: virtual, physical, and cognitive. Virtual models are those that exist in virtual space, e.g. CAD models, CFD and FEA simulations, etc. Physical models are those that exist in the physical space of the real-world, e.g. clay models, cardboard and sticky tape, etc. Cognitive models are then the mental models of the product(s) as conceived by the designer, and as communicated to others through the generation of physical and virtual models. In this description of design, the roles of the physical, virtual and cognitive models are equally important, and yet there is no formal framework or model of the design process that embodies such a framing or acknowledges the process(es) of switching or transitioning between models. For example, consider the case where a designer generates a rough cognitive model or concept, which they then explore and evaluate through making physical and virtual

\footnotetext{
${ }^{1}$ https://www.open.edu/openlearn/science-maths-technology/design-innovation/design/content-section-1.4. Last visited 2019-11-19.
} 
representations of said model. The concept evolves towards a final design as it is evaluated in physical (e.g. strength test), virtual (e.g. CFD), cognitive realms (e.g. user studies). Switching between models allows the designer to optimise the learning through design iterations as they select the most appropriate medium for the evaluation method required. See Figure 1.

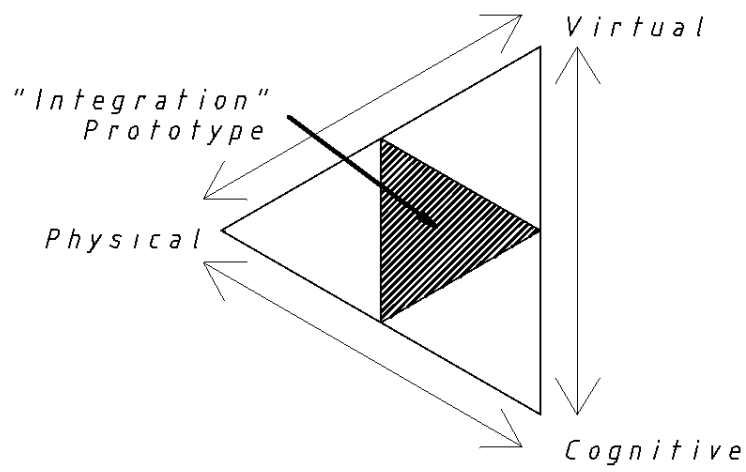

Figure 1. A framework of design as physical, visual and cognitive models; the transitions between the three models; and two or more models working simultaneously

Not only does this framing of design highlight the designer as a cornerstone of the design process, it also allows for insights into the design process that could ultimately lead to improvements in the field. Through improving the transitions between models, the overall design process can arguably be improved, i.e. the quicker a designer can virtually/physically realise a concept, the quicker that concept can be communicated and evaluated.

Given that trends are pushing towards the integration of physical and virtual with a passive removal of the designer from the devpsign process, it is timely and important that we model and clarify the role the designer must play in the future of design processes. This paper therefore aims to contribute to the field of design science by proposing the framing of design in terms of physical, virtual and cognitive models, and the transitions between them. A high-level overview of the model is given as a first attempt to explore the concept while both cementing the designer in the design process and providing the means to improve the design/design process. This is achieved through grounding the framework in existing design tools, techniques, and literature, and aligning the framework with seminal literature in the design science field. Section 2 provides a brief background in discussing the role of physical, virtual, and cognitive models in design, Section 3 then presents the framing of design in terms of physical, virtual, and cognitive models in more detail and the delivery of a form of the integration prototype earlier in the design process. Section 3.1 focuses on the processes of switching between models and highlights current research efforts in this area. Section 3.2 discusses how the three types of model can work in parallel to aid design, again with a focus on literature. Finally, Section 4 aligns this framing within design science.

\section{Models in design}

Working on the principle that design involves the creation, evaluation, and evolution of three types of models - physical, virtual, and cognitive - this section describes design in terms of each model and provides relevant background and terminology used throughout the rest of this paper.

\subsection{Physical design and physical models}

Physical design and physical models refer to the use of materials and manufacturing processes such as cardboard and sticky tape, clay, and 3D printing. Physical design is the process of the designer engaged in making: the act of taking raw materials, such as cardboard and sticky tape, and transforming them into a physical embodiment of the design, as well as paper-based sketching and note-taking. McAlpine et al. (2017) show the importance of physical paper logbooks in engineering design and (Brereton and McGarry, 2000; Coutts et al., 2019; Jang and Schunn, 2012; Subrahmanian et al., 2003) are all examples of literature that show the importance of physical models in design. The physical embodiment of a design is shown to improve communication, understanding, and evaluation. 
The argument against physical design is the time, skill, and resources required make complex physical models. This is even true with the efforts to de-skill the act of making through machines such as additive manufacture. Physical models are then important to the design process but take time and effort to make, arguably the reason why the design field has seen such a growth in virtual design and virtual models.

\subsection{Virtual design and virtual models}

While this paper discusses this topic in terms of designs and models, within the context of virtual environments, these form the wider field of virtual prototyping. Zorriassatine et al. (Zorriassatine et al., 2003) describes virtual prototyping as constituting of three types of activity: mechanical design (e.g. sketching, two/three-dimensional drafting); shape design and styling (e.g. complex free-form curves); and analysis and simulation (e.g. design optimisation, CFD, FEA). Virtual models are computer generated geometric representations of the design, and virtual design is the activity of creating and evolving that design through virtual techniques (design, styling, simulation and optimisation). These virtual techniques are enabled through software packages: CAD for modifying geometry, CAM for converting geometry into machine instructions, etc. In addition to these, however, the virtual design also encompasses the office suites, databases, email clients, and internet search engines which all play a role in the research, creation, management of virtual documents.

Cecil and Kanchanapiboon (Cecil and Kanchanapiboon, 2007) describe the benefits of virtual prototyping as earlier detection of design and manufacturing problems; support of concurrent engineering practices; and the reduction in the lead time for both manufacture and new product development. There are, however, disadvantages to virtual prototyping. There are a number of studies that highlight the importance of physical prototyping (Brereton and McGarry, 2000; Coutts et al., 2019; Jang and Schunn, 2012), showing that the projects that perform physical prototyping activities generate better designs than those that rely on virtual techniques. So, while virtual prototyping has its advantages, designers should be wary of designing as a pure virtual activity.

\subsection{Cognitive design and cognitive models}

Cognitive design effectively consists of the activities of ideation, creativity, and the role of the human mind in the evaluation and the decision-making process. Whilst each of these areas is the focus of many research papers, they are often seen as less tangible than physical and virtual models and, hence, more difficult to study and quantify. Jansson et al. (Jansson et al., 1993) examined the role of cognition in design and identified three cognitive processes: identification (understanding and representing the problem); synthesis (reasoning and evaluating the design as it evolves); and evaluation (testing the design). In other research, Brereton and McGarry (Brereton and McGarry, 2000) identified the importance of the physical boundary objects in design communication. Design involves a number of stakeholders, each likely to have their own cognitive model of the design. The physical embodiment of one's cognitive model better enables the communication of that model.

\section{Design as the pathways between models}

Similarly, in order to understand the role of physical, virtual and cognitive models, there is also a need to understand the pathways between models, i.e. how the design transitions from one model to another. This section explores the framework in terms of the three means of working with models in design: seamlessly switching between each model; working in all three models in parallel; and integrating two or more models to deliver a form of the integration prototype (Houde and Hill, 1997).

\subsection{Seamless model transitioning in the design process}

This paper has shown that, as standard practice, physical, virtual, and cognitive models are used throughout the design process, including during concept generation. During these earlier phases, the transition between physical, virtual, and cognitive models takes place and is arguably an important aspect of design (see Figure 1). As such, the quicker and more accurately one can transition between models, the quicker the design process. 
Consider the journey from converting a customer email into a list of requirements and producing the first looks-like prototype. The virtual email is read and understood, converting it to a cognitive prescriptive model in the mind of the designer. This is then formally documented, for example, in a virtual spreadsheet. Through ideation activities, the prescriptive requirements list is converted into a concept (cognitive model) that is then virtually embodied in CAD, before being 3D printed (physical model).

Any delay in those transitions between models delays the overall design process. Any reduction in the accuracy of the model introduces error and ambiguity, which again can delay the design process. In addition to time, there are cognitive loads involved in these transitions, for example the acts of: interpreting a user requirement; interacting with a CAD software package; and making a physical prototype. A focus on the optimisation of switching between models therefore has the potential to improve the design process.

\subsubsection{Transitioning between physical and virtual models}

Transitioning between physical and virtual models is effectively the act of manufacturing/making and some form of metrology/virtual capture (Jones et al., 2019). In terms of manufacturing/making, the high cost of tooling and certain manufacturing processes are prohibitive to their use in the making of prototypes and so there is a focus on hand-making techniques (cardboard, sticky tape, foam, clay, etc.) and the ever-increasing use of low cost additive manufacturing. Robust low cost metrology methods are the subject of current research, with techniques based on photography beginning to show promise (Dickins et al., 2018; Lavecchia et al., 2018; Sims-Waterhouse et al., 2017). Hattab and Taubin (Hattab and Taubin, 2015) discuss the combined use of metrology and additive manufacturing, describing the synchronisation of virtual CAD models and physical printed models using three-dimensional scanner. A physical model is captured in the form of a virtual point cloud and the corresponding virtual CAD model is automatically updated to reflect the changed state of the physical model.

This act of synchronisation leads on to the use of the digital twin in early stage design, as proposed by Jones et al. (Jones et al., 2019). Later in the product life-cycle, the digital twin is expected to optimise performance, and extend life-cycles as real-world data is fed from a physical entity to a virtual-butidentical entity. The combination of real-world data and high-fidelity models allows virtual techniques of simulation, modelling, and optimisation to effectively run virtual 'what-if' scenarios using real-time data. The output of these analyses can be used to modify the operation of the physical entity. The use of the digital twin paradigm between the physical and virtual models effectively removes the effort required of the designer to transition between physical and virtual models. However, while describing potential benefits of the approach (computational offloading, revision control, data driven design), Jones et al. (2019) also highlight tshat there is still some way to go in terms of providing a technology solution to delivering this.

Whilst these first two examples rely on a more traditional make-modify-scan-update iteration, there are other novel approaches to the transition between physical and virtual model transitioning. Jones et al. (2018) present 3D-printed artefacts that represent physical user interface controls (electronic switches) and are marked with fiducial markers. The designer incorporates the artefacts into the prototype form, and upon scanning, the fiducial markers are recognised and interpreted as the actual electronic input devices. This removes the need to manually update the CAD model with off-the-shelf components and their positions. Wessely et al. (Wessely et al., 2018) present ShapeMe, a shape-aware material (paper with electrical strips) that is able to detect where the designer cuts the paper. Cutting the sheet of ShapeMe paper automatically updates the corresponding CAD counterpart. Harrison et al.(Harrison et al., 2015) present an exploratory study into an approach to capture motion in kinematic designs, using a generative approach to understand the relationship between shape, structure and motion. These three examples show how, when considering the task of switching between physical and virtual models, there are a range of standard and novel techniques being experimented with that should ultimately lead to improvements and automations in the processes.

\subsubsection{Transitioning between physical and cognitive models}

Transitioning between physical and cognitive models in design can be divided into three types of actions: the act of embodying a cognitive model; the act of communicating a cognitive model to 
another individual or individuals; and the act of evaluating a physical model against one's cognitive model.

The act of embodying a cognitive model is the act of making, typically using materials such as cardboard, sticky tape, foam, etc. and this is heavily reliant on the skill set of the designer. Ferguson (Ferguson, 1992, 1993) argues for the use of physical models in design, stating that engineering students have become too reliant on virtual models and techniques, and that this misses important tactile learning opportunities. However, whether this translates to professional designers rather than students is unknown. One could argue that, through the act of design drafting and onset of desktop additive manufacturing technology, there has been a reduction in the required physical hands-on skill set. The skill set here being the designer translating their cognitive model to paper (sketching) or virtual models (CAD for example) rather than directly to a physical three-dimensional model. As stated in Section 2.1, there are a number of published papers that show the importance of the physical model in design (Brereton and McGarry, 2000; Coutts et al., 2019; Jang and Schunn, 2012), whether the designer/design is improved with tactile hands-on making is unknown at this moment.

The act of communicating a design is the act of creating and/or updating another individual's cognitive model. Literature here shows the importance of boundary objects in communication. Having a shared entity around which to structure discussions ensures that all stakeholder cognitive models remain aligned. While one could argue that it could never be certain that two individual cognitive models are aligned, the boundary object acts as a mediator in the communication, something upon which all parties can agree.

Design evaluation is partly the act of comparing a design against the design requirements, and occasionally the act of generating and/or comparing end-user cognitive models against the design. This involves providing an individual with the physical design and somehow capturing responses, e.g. questionnaires. These forms of measurement capture not only whether a design functions as intended, but also whether the design behaves as the end-user would expect. This provides assurance that the designer has designed appropriately, that a market exists, and that assumptions made in the design process were correct.

\subsubsection{Transitioning between virtual and cognitive models}

Transitioning between virtual and cognitive models is the act of virtually capturing a design on a computer, and cognitive learning, understanding and evaluating using a virtual representation of the design. The virtual capture of design can occur in a number of ways across the design process: from a collection of inspirational images aimed at inspiring creativity and ideation, to spreadsheets of requirements (design prescription), and CAD and CAM software packages. The cognitive learning, understanding, and evaluation of virtual designs is the study of reports, bill-of-materials, rendered CAD models, and raw CAD models, using technology ranging from a desktop computer, to immersive virtual and augmented reality.

While these seem simple enough, both the virtual-to-cognitive and cognitive-to-virtual techniques are heavily dependent on the field of Human-Computer Interfaces (HCI). The ability to judge a design can be more dependent on the technology interface being used rather than the design itself. For example, Autodesk Research spend considerable resources on the improvement of next-generation graphical user interfaces. McCrae et al. (McCrae et al., 2010) present work exploring the user's ability to navigate within multi-scale three-dimensional environments; Vanacken et al. (Vanacken et al., 2009) present research on selection techniques in three-dimensional environments; Grossman et al. (Grossman et al., 2002) present the use of digital tape to draw three-dimensional curves; Khan et al. (Khan et al., 2008) present ViewCube, a means of switching between present views in threedimensional space; and back in 1997, Kurtenbach et al. (Kurtenbach et al., 1997) were addressing the challenges of interacting with design on tablet devices.

Outside of the more traditional graphical user interface research, design research is increasingly experimenting with virtual and augmented reality technologies. Virtual reality immerses the user in a virtual environment, while augmented reality superimposes virtual representations over the user's physical environment. Berg et al. (Berg and Vance, 2017) surveyed a number of engineering industry applications for virtual reality and found, amongst other things, that given the ability to view a fully 
rendered life-size model, virtual reality is a useful tool in decision making. Counter to this, however, Coutts et al. (Coutts et al., 2019) show that physical prototyping outperforms both virtual and augmented reality techniques, with augmented reality performing the worst.

One key question in the virtual-to-cognitive and cognitive-to-virtual transitions is the suitability of offthe-shelf software packages when used in design activities. Does, for example, a traditional text-based search engine provide the most appropriate means of finding design information, or would a modelbased information navigation (Jones et al., 2015) system be more suitable? Does your favourite spreadsheet software or email client inspire creativity and ideation, or does it hinder the processes? Key to a seamless transition between cognitive and virtual models is minimising the user's cognitive load, something that should underpin all graphical user interfaces (Rogers et al., 2011). In addition to minimising cognitive loads, should software interfaces be re-designed to aid the designer? Often the physical environments in which designers work are filled with their products and physical models of various stages of design, interesting and inspiring forms aimed at encouraging acts of creativity and ideation. Where are the software equivalents?

\subsection{Physical, virtual, and cognitive models in parallel}

This section examines working in two or more of the models in parallel, indicated in the shaded region in Figure 1. Wendrich (Wendrich, 2018) explores the physical, virtual, and cognitive models working in parallel, or as named in the paper, a hybrid approach to prototyping. The system presented combined tangible materials, virtual display units, metrology methods, and the designer such that the designer can prototype in a physical medium and be presented with real-time information and virtual support and/or feedback from other stakeholders. The system, IEK-framework: IEK-Spraction (Intuition/Intention-Experience-Knowledge) aims to remove boundaries between the physical, virtual, and cognitive models such that the designer is simply free to design.

In earlier work, Wendrich (Wendrich, 2010) also calls for tangible interfaces for CAD, the idea that the designer can be free to design in a physical medium, and have that process automatically captured and translated into a virtual model. The Virtual Design Assistant (VDA) aims to "...pair them with, rather than to confront them with the digital modelling constraints and perceived affordances". This is a slightly different take on these models working in parallel in that the virtual is seen as almost working as a virtual assistant alongside the designer, rather than something the designer must actively engage with.

\subsection{Integrating the three models of design}

The Houde et al. (Houde and Hill, 1997) classification of prototypes into role, implementation, and look and feel is one familiar to many design researchers. 'Role' represents the purpose of a design, 'implementation' represents the functional working of a design, and 'look and feel' represents the sensory experience of interacting with the prototype. Through the integration of these classes, Houde et al. also demonstrate the formation of the 'integration prototype', a prototype with a form through which the user experiences a true representation of a complete design and the complete design can be evaluated. Framing design in terms of physical, virtual and cognitive models also allows the generation of a form of an integration prototype.

Key to integration prototypes in is the construction of physical prototypes that work, look and feel, and perform the role that they are intended for. Key to integration prototypes in Figure 1, however, is bringing together the physical, virtual, and cognitive models such that the three models operate in unison, to generate prototypes that appear to work, look and feel, and perform the role as outlined by the design. Effectively, the virtual model can be used to augment the physical model such that the cognitive model can be updated with what appears to be a complete design.

As an example, consider a prototype mobile phone. The casing and screen can be additively manufactured and weighted such that it feels like a working phone, with no working inner circuitry. An augmented reality system would then overlay the operating system on the physical model, and capture and respond to user interactions. From the user perspective, other than the wearing of an augmented reality headset, the experience would be close to a fully functional physical prototype. This 
achieves the integration prototype without having to construct the physical inner circuitry, screen, buttons, etc.

This is not the first time that such an approach to prototyping has been proposed. The concept of augmented prototyping has seen some interest in the last few years as has the use of haptic feedback in virtual prototyping. Augmented prototyping combines a physical and virtual model such to mimic a more complete prototype. Verlinden et al.'s (Verlinden et al., 2003) Workbench for Augmented Rapid Prototyping (WARP) is a system that projects images over physical models, while Giunta et al. (Giunta et al., 2019) present a study comparing the technique to screen-based three-dimensional models, finding that the AR approach could improve communication. Park et al. (Park and Moon, 2013) present a few use-cases such as game controller and mobile phones, where, through the use of fiducial markers, physical artefacts are augmented to look and function like the integration prototypes. The use of haptics in virtual reality is aiming to achieve the same experience from within a virtual environment. Volkov and Vance (Volkov and Vance, 2001) evaluated the use of haptics in design evaluation, finding that users spent significantly less time evaluating and preferred the use of haptic feedback over not using it while achieving no significant differences in evaluation performance between the two systems.

Both augmented prototyping and haptic feedback in virtual reality show examples where the physical and virtual are combined to provide a fuller experience in prototype design. That fuller experience being the representation of the integration prototype. As a warning of the use of virtual without the physical, Coutts et al. (Coutts et al., 2019) presented an evaluation of different prototyping techniques (physical, CAD, virtual reality and augmented reality) and found augmented reality performed the worst. The augmented reality set-up lacked a physical component, however - something highlighted by Coutts et al. in their conclusion.

The importance of the physical in this approach calls for feels-like prototypes, achieved through physical models that can mimic the weight, inertia, and surface properties (in terms of touch) of the final design. There is work ongoing at the University of Bristol into appropriately replicating weight and inertia in additively manufactured components. There is still work required in replicating the surface properties using rapid prototyping techniques, for example, replicating the feel of a mobile phone screen using a $3 \mathrm{D}$ printer.

\section{Alignment with design science}

The previous three sections explored and proposed the framing of design in terms of physical, cognitive, and virtual models; working in parallel with two or more models; and the transitions between models. This section now aligns the framing with some of the relevant and seminal works of design science, starting with the work of Steinert and Leifer and wayfaring.

Wayfaring in design as proposed by Steinert and Leifer (Steinert and Leifer, 2012) frames design an exploration that leads to a design solution rather than a prescribed plan to innovate new ideas. In wayfaring, design is said to involve diverse multidisciplinary teams who make strides towards perceived design solutions, steps are divergent and involve the making and evaluation of prototypes. The results of evaluation are then used to direct the next step. With every step, the designers learn more about the problem/solution and iterate the design towards the final design. Key to the success of wayfaring is the speed of iteration and the agility in steering the next step. Quicker iterations produce quicker learning, and an agile nature aims to derestrict the direction in which the design evolves. Translating wayfaring in terms of physical, virtual, and cognitive models, each step in the wayfaring starts with a cognitive model that is embodied either physically or virtually. Those embodied models are then evaluated, knowledge is gained, updating the cognitive model. That new cognitive model forms the basis of the next step in the exploration.

The CK theory (Hatchuel and Weil, 2003) frames design within the knowledge spaces (K) and concept spaces (C). Design involves the four operation: $K \rightarrow C, C \rightarrow K, C \rightarrow C$, and $K \rightarrow K$. These make up the design square. $\mathrm{K} \rightarrow \mathrm{C}$ operations add/subtract concepts from the concept space using knowledge. $\mathrm{C} \rightarrow \mathrm{K}$ operations seek knowledge to update the concept space. $\mathrm{C} \rightarrow \mathrm{C}$ operations are the means of controlling whether concepts are included/excluded. $\mathrm{K} \rightarrow \mathrm{K}$ operations are the processes of gaining knowledge from existing knowledge. CK theory can be mapped to physical, virtual, and cognitive models in terms of the pathways that the design takes between models. Virtual $\rightarrow$ physical is the 
process of CAM, and physical $\rightarrow$ virtual is the process of metrology for example. The transitions between physical and virtual do not relate to CK theory given they do not include the cognitive model. The pathways physical $\rightarrow$ cognitive and virtual $\rightarrow$ cognitive are both examples of $\mathrm{K} \rightarrow \mathrm{C}$ operations, as knowledge is gained from embodied designs and used to update the concept space. Cognitive $\rightarrow$ virtual and cognitive $\rightarrow$ physical are both examples of $\mathrm{C} \rightarrow \mathrm{K}$ operations, where elements of concepts are embodied to evaluate specific elements of the concept.

Schön's contribution on design as a reflective conversation with materials (Schön, 1992), is framed in terms of the designer "seeing, moving, then seeing again". Effectively, the designer examines a design and reflects on it before taking some form of action in response to the reflection, i.e. evolving the design. In this seminal paper, Schön describes the processes of architects re-designing classrooms, showing how the designers switched between "seeing" the current design, "drawing" a design solution, and "seeing" that new design solution on the page. Schön's examples show the pathway between cognitive and physical models (paper sketches). These transitions allow for "seeing patterns" in the physical realisation (updating and evolving the cognitive model). The updated cognitive model is then used to update the physical model, and so the cycle continues. Schön continues through a discussion on how the type of material using the physical model can affect design decisions, effectively showing the medium of the physical models affects the design. Schön's paper is attempting to lay the foundations for artificial intelligent design, and concludes that to be successful, artificial intelligence will need to simulate a range of the designer actions. This is more akin to replacing the designer with a virtual one, rather than designing them out. It is interesting that in Schön's concluding comments, it is stated that the role of artificial intelligence in design is more promising in the field of design assistance.

Goldschmidt and Porter (Goldschmidt, 2004), frame design in terms of private processes and public images. With design representations taking on different purposes, modularity, media (paper sketched, $\mathrm{CAD}$, etc.), and level of abstraction, as well as both internal (cognitive) and external (physical and virtual), Goldschmidt and Porter discuss the importance of designing with the "right" media. All forms of media have benefits to the design and, as such, should be made accessible to the designer. This simply reaffirms the framing of design in terms of physical, virtual, and cognitive models. The seamless switching between and the combination of models allows the designer to design in the medium most suitable to their needs and to adapt as those needs change along with the evolving prototype. As an example of this, McAlpine et al. (McAlpine et al., 2017) discuss the use of the engineer's logbook in mediators in engineering design, finding that the logbook acts as mediators through facilitating cognition and creativity; gathering and collation information; and staging and transformation of information. The engineer's logbook is a physical item and a means of supporting and informing the cognitive model. Through activities of sketching and note-taking, both formally and informally, the physical logbook acts as a means of rapid cognitive $\rightarrow$ physical and physical $\rightarrow$ cognitive transitions that in themselves facilitate CK operations and wayfaring.

Gero's function-behaviour-structure framework (Gero, 1990; Gero and Kannengiesser, 2004) describes design in terms of function (the purpose of a design artefact), behaviour (the attributes of a design prototypes derived from its structure), and structure (derived from the prototype components and assembled components). The framework brakes down design in terms of requirements (R), descriptions (D), function (F), structure (S), and behaviour split into expected (Be), structural (Bs) and actual (Ba). Furthermore, 8 design processes are described: formulation, synthesis, analysis, evaluation, documentation, reformulation type 1 , reformulation type 2 , and reformulation type 3 . See (Gero, 1990) for formal descriptions of each of these. The function-behaviour-structure framework does not distinguish between physical and virtual prototypes and arguably it does not need to: in aligning this with the types of models used in design and at a high level, it does result in the physical and virtual models being somewhat interchangeable. There are still, however, elements of both frameworks that map. Taking behaviour as an example, one could argue that the expected behaviour is the cognitive model, the structural behaviour is the physical model and the actual behaviour is then a trade-off between the cognitive model and what is physically possible. Function-behaviour-structure describes this as reformulation type 2 - the expected behaviour is updated as structure is interpreted. Similarly, the processes of formulation describes how requirements inform function and that function informs expected behaviour. This is arguably the pathway from requirements to a cognitive model. 
The process of documentation could then also be the pathway from physical-to-virtual as the actual final physical design is captured and formalised for manufacture.

The function-behaviour-structure framework is rich in detail and within the limitations of this paper it is not possible to present a complete alignment, however this discussion does show that the two frameworks can align. Similarly, there are other seminal works that were not able to be covered here and will require further work, for example Menold et al. Prototyping for X (Menold et al., 2017).

\section{Conclusion}

During design, designers employ three types of model: physical, virtual and cognitive. The role and contribution of each to the designer and design process is documented in existing literature although it is somewhat fragmented in nature. It is contended that this fragmentation and lack of integrated or holistic understanding poses a major risk due to the omission of the cognitive models in many emerging paradigms, such as digital twins.

This paper begins to address this gap though the proposition that the design process be framed in terms of physical, virtual and cognitive models, and the pathways between them. Not only does this framework highlight the importance of the designer and their cognitive models, but it also shows how improving the pathways between models can lead to improvements in design. This paper explores this framework, showing existing literature in each of the models and pathways, and aligns it with some of the key seminal works of design science. A formal description of the framework is not presented. This requires further work and alignment with current design science, such as function-behaviour-structure frameworks and prototyping for $\mathrm{X}$. To achieve this, the following questions need addressing:

1. What do the transitions between models look like in 'real-world' design projects?

2. Is it possible to prescribe the point in the design process when each model is appropriate?

3. How would one evaluate a formal model?

Through answering these questions it will be possible to both produce a formal framework for design based on the types of models that designers use, the transitions between them. This places the designer on equal footing with the virtual and physical models that are the main focus of other research efforts. Similarly, evaluating the formal framework would then provide confidence to both design researchers and practitioners that approaching design in such a way could ultimately benefit us all.

\section{Acknowledgements}

The work reported in this paper was undertaken as part of the "Improving the product development process through integrated revision control and twinning of digital-physical models during prototyping" at the University of Bristol. The project is funded by the Engineering and Physical Sciences Research Council (EPSRC) Grant EP/R032696/1.

\section{References}

Berg, L.P. and Vance, J.M. (2017), "Industry use of virtual reality in product design and manufacturing: a survey", Virtual Reality, Vol. 21 No. 1, pp. 1-17.

Brereton, M. and McGarry, B. (2000), An observational study of how objects support engineering design thinking and communication: implications for the design of tangible media, pp. 217-224.

Cecil, J. and Kanchanapiboon, A. (2007), "Virtual engineering approaches in product and process design", The International Journal of Advanced Manufacturing Technology, Vol. 31 No. 9-10, pp. 846-856.

Coutts, E.R., Wodehouse, A. and Robertson, J. (2019), A comparison of contemporary prototyping methods.

Dickins, A. et al. (2018), "Design of a multi-sensor in-situ inspection system for additive manufacturing", Proc. ASPE/Euspen Advancing Precision in Additive Manufacturing, pp. 242-248.

Ferguson, E.S. (1992), Engineering and the Mind's Eye, MIT press.

Ferguson, E.S. (1993), "How engineers lose touch", Invention \& Technology, Vol. 8 No. 3, pp. 16-24.

Gero, J.S. (1990), "Design prototypes: a knowledge representation schema for design", AI Magazine, Vol. 11 No. 4, p. 26.

Gero, J.S. and Kannengiesser, U. (2004), "The situated function-behavior-structure framework", Design Studies.

Giunta, L. et al. (2019), "Investigating the Impact of Spatial Augmented Reality on Communication between Design Session Participants-A Pilot Study".

Goldschmidt, G. (2004), "Design representation: Private process, public image”, In Design representation. Springer. 
Goudswaard, M., Hicks, B. and Nassehi, A. (2018), "Democratising the design of 3D printed functional components through a hybrid virtual-physical design methodology", Procedia CIRP, Vol. 78, pp. 394-399.

Grossman, T. et al. (2002), Creating principal 3D curves with digital tape drawing, pp. 121-128.

Harrison, L., Earl, C. and Eckert, C. (2015), "Exploratory making: Shape, structure and motion", Design Studies.

Hatchuel, A. and Weil, B. (2003), "A new approach of innovative Design: an introduction to CK theory", DS 31: Proceedings of ICED 03, the 14th International Conference on Engineering Design, Stockholm.

Hattab, A. and Taubin, G. (2015), 3D modeling by scanning physical modifications, pp. 25-32.

Houde, S. and Hill, C. (1997). "What do prototypes prototype?", In Handbook of human-computer interaction.

Jang, J. and Schunn, C.D. (2012), "Physical design tools support and hinder innovative engineering design", Journal of Mechanical Design, Vol. 134 No. 4, p. 41001.

Jansson, D.G., Condoor, S.S. and Brock, H.R. (1993). "Cognition in design: Viewing the hidden side of the design process", Environment and Planning B: Planning and Design, Vol. 20 No. 3, pp. 257-271.

Jones, D.E. et al. (2015), "A Strategy for Artefact-Based Information Navigation in Large Engineering Organisations", DS 80-10 Proceedings of the 20th International Conference on Engineering Design (ICED 15), 27-30 July 2015.

Jones, D.E. et al. (2019), "Early Stage Digital Twins for Early Stage Engineering Design", Proceedings of the Design Society: International Conference on Engineering Design, Vol. 1 No. 1.

Jones, M.D. et al. (2018). PHUI-kit: Interface Layout and Fabrication on Curved 3D Printed Objects. p. 110.

Khan, A. et al. (2008), ViewCube: a 3D orientation indicator and controller, pp. 17-25.

Kurtenbach, G. et al. (1997), The design of a GUI paradigm based on tablets, two-hands, and transparency, pp. 35-42.

Lavecchia, F., Guerra, M.G. and Galantucci, L.M. (2018), "Performance verification of a photogrammetric scanning system for micro-parts using a three-dimensional artifact: adjustment and calibration", The International Journal of Advanced Manufacturing Technology, Vol. 96 No. 9-12, pp. 4267-4279.

McAlpine, H., Cash, P. and Hicks, B. (2017), "The role of logbooks as mediators of engineering design work", Design Studies, Vol. 48, pp. 1-29.

McCrae, J. et al. (2010), Exploring the design space of multiscale 3D orientation. pp. 81-88.

Menold, J., Jablokow, K. and Simpson, T. (2017), "Prototype for X (PFX): A holistic framework for structuring prototyping methods to support engineering design", Design Studies, Vol. 50, No. 70-112.

Park, H. and Moon, H.-C. (2013), "Design evaluation of information appliances using augmented reality-based tangible interaction", Computers in Industry, Vol. 64 No. 7, pp. 854-868.

Rogers, Y., Sharp, H. and Preece, J. (2011). Interaction design: beyond human-computer interaction. John Wiley \& Sons Chichester.

Schön, D. A. (1992), "Designing as reflective conversation with the materials of a design situation", KnowledgeBased Systems, Vol. 5 No. 1, pp. 3-14.

Shea, K., Aish, R. and Gourtovaia, M. (2005), "Towards integrated performance-driven generative design tools", Automation in Construction, Vol. 14 No. 2, pp. 253-264.

Sims-Waterhouse, D. et al. (2017), "Experimental comparison of photogrammetry for additive manufactured parts with and without laser speckle projection", Optical Measurement Systems for Industrial Inspection X, 10329, 103290W.

Steinert, M. and Leifer, L.J. (2012), "Finding One's Way": Re-Discovering a Hunter-Gatherer Model based on Wayfaring", International Journal of Engineering Education, Vol. 28 No. 2, p. 251.

Subrahmanian, E. et al. (2003), "Boundary objects and prototypes at the interfaces of engineering design", Computer Supported Cooperative Work.

Vanacken, L., Grossman, T. and Coninx, K. (2009), "Multimodal selection techniques for dense and occluded 3D virtual environments", International Journal of Human-Computer Studies, Vol. 67 No. 3, pp. $237-255$.

Verlinden, J.C. et al. (2003), Development of a flexible augmented prototyping system.

Volkov, S. and Vance, J.M. (2001), "Effectiveness of haptic sensation for the evaluation of virtual prototypes", J. Comput. Inf. Sci. Eng., Vol. 1 No. 2, pp. 123-128.

Wendrich, R.E. (2010), "Raw shaping form finding: Tacit tangible CAD", Computer-Aided Design and Applications, Vol. 7 No. 4, pp. 505-531.

Wendrich, R.E. (2018), "Multiple Modalities, Sensoriums, Experiences in Blended Spaces with Toolness and Tools for Conceptual Design Engineering”, ASME 2018 International Design Engineering Technical Conferences and Computers and Information in Engineering Conference.

Wessely, M., Tsandilas, T. and Mackay, W.E. (2018), Shape-Aware Material: Interactive Fabrication with ShapeMe. pp. 127-139.

Zorriassatine, F. et al. (2003), "A survey of virtual prototyping techniques for mechanical product development", Proceedings of the Institution of Mechanical Engineers, Part B: Journal of Engineering Manufacture, Vol. 217 No. 4, pp. 513-530. 